\title{
An atmospheric electrical method to determine the eddy diffusion coefficient
}

\author{
M N KulKARNi* and A K KAMRA** \\ Indian Institute of Tropical Meteorology, Dr. Homi Bhaba Road, Pune 411 008, India. \\ *e-mail: mnkulk2005@gmail.com \\ **e-mail: kamra@tropmet.res.in
}

The ion-aerosol balance equations are solved to get the profiles of atmospheric electric parameters over the ground surface in an aerosol-rich environment under the conditions of surface radioactivity. Combining the earlier results for low aerosol concentrations and the present results for high aerosol concentrations, a relation is obtained between the average value of atmospheric electric space charge in the lowest $\sim 2 \mathrm{~m}$, the surface electric field and eddy diffusivity/aerosol concentration. The values of eddy diffusivity estimated from this method using some earlier measurements of space charge and surface electric field are in reasonably good agreement with those calculated from other standard methods using meteorological or electrical variables.

\section{Introduction}

Hoppel and Gathman (1971) showed that it is possible to determine eddy diffusivity over the open-ocean from atmospheric electric measurements. In the method suggested by them, eddy diffusivity can be determined either from profiles of average electric field or from average values of space charge, electric field and aerosol concentration near the sea surface. Hoppel and Gathman (1972) later made such measurements off the east coast of Barbados and found good agreement of the eddy diffusivity estimates made from their atmospheric electric method with those made from the bulk aerodynamic method.

As an advantage of atmospheric electric method over eddy correlation method for the estimation of fluxes, Markson and Sedlacek (1981) mention that as the flux of electric charge (called the eddy current) is due to the positive space charge in the electrode layer and the charge density in this layer is proportional to the total atmospheric current, it can be estimated using the scale analysis method (Willett 1978) from simple dc measurements of the electric field and conductivity from a light aircraft while the latter method requires expensive arrangements including inertial navigation system, large computer power and large aircraft with gust probes. Their aircraft measurements off the California coast show good correlation of the surface layer eddy current peak values with the surface water vapour flux and the convective mixed layer velocity. Also, they find excellent signal-to-noise ratio in the case of atmospheric electrical technique over that of eddy correlation technique since in the case of vertical velocity derived measurements, the corrections made due to fluctuations in the vertical velocity are so large that they may be much greater than the vertical velocity itself. For the electric field data, these corrections are so small that they need not even be calculated. Since, space charge undergoes decay in time and is a passive scalar, it does not get well mixed in PBL and gives rise to gradients, which can be measured easily. This allows accurate determination of the passive scalar eddy diffusion coefficient, $K$ which was difficult to measure in the past. Many numerical models use this turbulent eddy diffusion coefficient $K$ to predict the dispersal of pollutants.

Keywords. Atmospheric electrical profiles; electrode layer; ion-aerosol balance equations. 
Kulkarni and Kamra (2001) (hereinafter referred as KK) solved the ion-balance equations including the effect of surface radioactivity in the presence of low aerosol concentrations and suggested that eddy diffusivity can be determined from the surface measurements of electric field and largeion space charge density for fixed values of total aerosol concentration. In this paper, we solve the ion-balance equations including the effect of surface radioactivity in the presence of high aerosol concentrations. Based on the present and earlier solutions of KK, we describe a method with which both the eddy diffusivity and aerosol concentration can be determined in a clean or polluted atmosphere. The estimates of eddy diffusivity made from some earlier measurements have been compared to the estimates obtained from the bulk aerodynamic and other methods.

\section{Model}

Recombination of ions and their attachment to aerosol particles are the two dominant processes which govern the dissipation of ions. In the presence of large aerosol concentrations however, recombination of ions can be neglected as compared to attachment of small ions to aerosol particles (Willett 1983). This can be verified using the scale analysis of ion-aerosol balance equations. Further, we can assume the average vertical velocity to be negligible since we are assuming uniform terrain with no orographical irregularities such as hills or valleys or multi-storied buildings to generate any standing waves. As production of turbulence due to shear always dominates that due to buoyancy near the surface even in a very unstable layer (Willett 1979), we can use eddy diffusion theory (K-theory) in our model equations. K-theory is appropriate for near neutral stability conditions, i.e., when vertical velocity is small or in condition of negligible buoyancy (Holton 1972; Stull 1988). Therefore, assuming that the average electric field is horizontally uniform, the average vertical wind is negligible and that the atmosphere is horizontally homogeneous, the steady state ion-aerosol balance equations over a flat surface can be written as:

$$
\begin{aligned}
& \frac{d J_{1}}{d z}=q-\beta_{0} n_{1} N_{0}-\beta_{1} n_{1} N_{2}, \\
& \frac{d J_{2}}{d z}=q-\beta_{0} n_{2} N_{0}-\beta_{1} n_{2} N_{1} \\
& \frac{d F_{1}}{d z}=\beta_{0} n_{1} N_{0}-\beta_{1} n_{2} N_{1}
\end{aligned}
$$

$$
\begin{aligned}
\frac{d F_{2}}{d z} & =\beta_{0} n_{2} N_{0}-\beta_{1} n_{1} N_{2}, \\
J_{1} & =k E n_{1}-K \frac{d n_{1}}{d z}, \\
J_{2} & =-k E n_{2}-K \frac{d n_{2}}{d z}, \\
F_{1} & =-\tau \frac{d N_{1}}{d z} \\
F_{2} & =-\tau \frac{d N_{2}}{d z}
\end{aligned}
$$

where $J_{1}$ and $J_{2}$ are the small ion fluxes due to positive and negative ions respectively in $\mathrm{m}^{-2} \mathrm{~s}^{-1}$, $F_{1}$ and $F_{2}$ are the fluxes due to positively and negatively charged aerosols respectively in $\mathrm{m}^{-2} \mathrm{~s}^{-1}, q$ is the rate of ionization in $\mathrm{m}^{-3} \mathrm{~s}^{-1}, n_{1}$ and $n_{2}$ are the concentrations of positive and negative small ions in $\mathrm{m}^{-3}$, and $N_{1}$ and $N_{2}$ are the concentrations of positively and negatively charged aerosols respectively in $\mathrm{m}^{-3} . N_{0}$ is the concentration of uncharged aerosol particles in $\mathrm{m}^{-3}$. $E$ is the atmospheric electric field in $\mathrm{V} \mathrm{m}^{-1} . \beta_{0}$ and $\beta_{1}$ are the attachment coefficients between small ions and electrically neutral aerosol particles and between small ions and aerosols of opposite polarity, respectively in $\mathrm{m}^{3} \mathrm{~s}^{-1}$. $K$ is the eddy diffusion coefficient for small ions in $\mathrm{m}^{2} \mathrm{~s}^{-1}, \tau$ is that for aerosol particles in $\mathrm{m}^{2} \mathrm{~s}^{-1}$ and $k$ is the mobility of small ions in $\mathrm{m}^{2} \mathrm{~V}^{-1} \mathrm{~s}^{-1}$. In the above equations, the variables represent their mean values averaged over time periods which are long compared to the largest scale eddies found in the atmosphere and during the periods when the conditions of steady state turbulence can be assumed. Following Hoppel (1967) and Hoppel and Gathman (1971), the vertical profiles of ionization $q$ and eddy diffusion coefficient $K$ and $\tau$ can be written as:

$$
\begin{aligned}
q(z) & =\left[Q_{0}+Q_{1} \exp (-2.362 z)\right] \\
K(z) & =\frac{\chi z+\gamma}{z+100}
\end{aligned}
$$

and

$$
\tau(z)=\frac{\chi z}{z+100}
$$

where $q(z)$ and $K(z)$ and $\tau(z)$ represent the values of $q, K$ and $\tau$ at height $z . Q_{0}=7 \times 10^{6} \mathrm{~m}^{-3} \mathrm{~s}^{-1}$ represents the ionization due to $\gamma$-radiations and cosmic radiations near the surface. The second 
term on the right hand side in the ionization profile represents the ionization due to trapped radon gas when $Q_{1}=80$ which is based on the theoretical calculations of Hoppel (1967) and measurements of Crozier and Biles (1966) and only due to $\beta$ radiation when $Q_{1}=4.8$ based on the measurements of Hess and O'Donnel (1951) and given by Hoppel (1969). In equation (10), $\chi$ is a mixing parameter in $\mathrm{m}^{2} \mathrm{~s}^{-1}$ and $\gamma$ is an ionic diffusion coefficient in $\mathrm{m}^{3} \mathrm{~s}^{-1}$ which is the measure of ionic diffusion and important in the micro-layer very near the surface.

From Poisson's equation, divergence of the electric field is proportional to the space charge density contained in that volume. Previous studies on atmospheric electrode layer (e.g., Hoppel and Gathman 1971; Kulkarni and Kamra 2001; Willett 1978) use the upper boundary conditions for the electrode effect modeling as both $\rho$ and $d \rho / d z$ vanish at the top of electrode layer. These studies show that the electrode layer positive space charge is maximum near the surface of the earth and decreases gradually as we go away from the surface. Also, the vertical gradient of it is strongest near the surface and its strength decreases as we go away from the surface. So, in the case of electrode layer, we find the behaviour of space charge density $\rho$ is directly proportional to that of its gradient $d \rho / d z$. Further, this can be justified mathematically as follows: while modeling electrode layer, the required upper boundary conditions are the same for space charge density $\rho$ and its gradient i.e., both vanish at the top of electrode layer irrespective of stability conditions. This is possible only when their respective percent rate of fall is the same at each level with respect to their surface values. i.e., when $\rho \alpha(d \rho / d z)$. So, the above assumption holds in all the stability conditions with respect to the given upper boundary conditions. Therefore, for the modeling of electrode layer, it is quite reasonable to replace $\rho$ in Poisson's equation with that of $d \rho$. Also, as we are considering the horizontally homogeneous situation, considering only the vertical component, we can write the following:

$$
d E=c_{1} d \rho,
$$

where $c_{1}$ is a constant of proportionality.

Integrating it,

$$
E=c_{1} \rho+c_{2},
$$

where $c_{2}$ is a constant of integration.

At $z=0$,

$$
E(0)=c_{1} \rho(0)+c_{2} .
$$

At $z=\infty$ i.e., when the asymptotic values of the parameters are reached,

$$
E(\infty)=c_{2}
$$

because $\rho(\infty)=0$.

Substituting (14) in (13) we get

$$
c_{1}=\frac{E(0)-E(\infty)}{\rho(0)}
$$

Therefore, at a height $z,(12)$ can be written as:

$$
E(z)=(E(0)-E(\infty)) \frac{\rho(z)}{\rho(0)}+E(\infty) .
$$

$E(z)$ can be calculated from (16) by using $n_{1}, n_{2}$, $N_{1}$ and $N_{2}$ obtained numerically from equations (1) to (4) to get $\rho(z)$.

The asymptotic values of electric field and aerosol concentration and the derived boundary conditions used here are as given in KK. For the sake of continuity we summarize them here:

As the derivatives of different parameters vanish when the asymptotic conditions are reached, from equations (1)-(4) we get

$$
N_{1}(\infty)=N_{2}(\infty)=N(\infty)=\frac{\beta_{0} Z}{2 \beta_{0}+\beta_{1}} .
$$

Due to omission of the recombination term in equations (1) and (2), we get asymptotic value of small ion concentration as:

$$
n_{1}(\infty)=n_{2}(\infty)=n(\infty)=\frac{q}{\beta Z},
$$

where $Z=N_{1}+N_{2}+N_{0}$ is the total aerosol concentration in the vertical column of electrode layer in $\mathrm{m}^{-3}$, and

$$
\beta=\frac{2 \beta_{0} \beta_{1}}{2 \beta_{0}+\beta_{1}} \mathrm{~m}^{3} \mathrm{~s}^{-1}
$$

where $\beta_{0}=1.4 \times 10^{-12} \mathrm{~m}^{3} \mathrm{~s}^{-1}, \beta_{1}=4 \times 10^{-12} \mathrm{~m}^{3} \mathrm{~s}^{-1}$, $e=1.6 \times 10^{-19} \mathrm{C}, \varepsilon_{0}=8.85 \times 10^{-12} \mathrm{~F} \mathrm{~m}^{-1}, \gamma=5 \times$ $10^{-4} \mathrm{~m}^{3} \mathrm{~s}^{-1}$ and $k=1.2 \times 10^{-4} \mathrm{~m}^{2} \mathrm{~V}^{-1} \mathrm{~s}^{-1}$. 
The total current density, $j$ in $\mathrm{A} \mathrm{m}^{-2}$, is constant with height and is given as:

$$
\begin{aligned}
\frac{j}{e}= & k E\left(n_{1}+n_{2}\right)-K(z)\left\{\frac{d n_{1}}{d z}-\frac{d n_{2}}{d z}\right\} \\
& -\tau(z)\left\{\frac{d N_{1}}{d z}-\frac{d N_{2}}{d z}\right\} \mathrm{m}^{-2} \mathrm{~s}^{-1} .
\end{aligned}
$$

The first term on the right hand side of (20) is due to the conduction of small ions, the second term is due to the convection of small ions, and the third term is due to the convection of charged aerosols.

Now, as discussed by Hoppel and Gathman (1971), ions are annihilated at the surface. So, $n_{1}(0)=0$ and $n_{2}(0)=0$. Further, since $\tau(0)=0$, there is no need to consider the source term for aerosols. On the other hand, at $z=0, K(0)$ is not 0 , and the continuity of current at the surface is maintained through the diffusion of space charge due to small ions. Also, from (3) and (7),

$$
\begin{aligned}
\frac{d F_{1}}{d z} & =-\tau(z) \frac{d^{2} N_{1}}{d z^{2}}-\frac{d \tau}{d z} \frac{d N_{1}}{d z} \\
& =\beta_{0} N_{0} n_{1}-\beta_{1} N_{1} n_{2} .
\end{aligned}
$$

Similarly, from (4) and (8),

$$
\begin{aligned}
\frac{d F_{2}}{d z} & =-\tau(z) \frac{d^{2} N_{2}}{d z^{2}}-\frac{d \tau}{d z} \frac{d N_{2}}{d z} \\
& =\beta_{0} N_{0} n_{2}-\beta_{1} N_{2} n_{1} .
\end{aligned}
$$

At $z=0$ it yields

$$
\left(\frac{d N_{1}}{d z}\right)_{z=0}=0
$$

Similarly,

$$
\left(\frac{d N_{2}}{d z}\right)_{z=0}=0
$$

Now, at the surface,

$$
\frac{j}{e}=-K(0)\left\{\frac{d n_{1}}{d z}-\frac{d n_{2}}{d z}\right\}_{0}
$$

The same current density at infinity, i.e., at the top of electrode layer in this case is given as:

$$
\frac{j}{e}=k E(\infty)\left[n_{1}(\infty)+n_{2}(\infty)\right] .
$$

Equating (25) to (26), and assuming $n_{1}(\infty)=$ $n_{2}(\infty)=n(\infty)$, we get

$$
E(\infty)=\frac{-K(0)}{2 k n(\infty)}\left\{\frac{d n_{1}}{d z}-\frac{d n_{2}}{d z}\right\}_{0} .
$$

There are now four unknown boundary conditions at $z=0$, namely, $N_{1}(0), N_{2}(0),\left(d n_{1} / d z\right)_{0}$, and $\left(d n_{2} / d z\right)_{0}$, which are to be chosen to satisfy the asymptotic boundary conditions given by (17), (18), and (27).

\section{Results and discussion}

\subsection{Profiles of atmospheric electric parameters in polluted atmosphere}

KK present the profiles of electric field and small ion and charged aerosol concentrations over a flat surface in relatively clean atmospheres with $Z=5 \times 10^{8}$ and $5 \times 10^{9} \mathrm{~m}^{-3}$. Here, we solve (1)-(4) and (16) to get such profiles in polluted atmosphere with $Z=10^{11} \mathrm{~m}^{-3}$. Results for $\chi=30$ are plotted in figure 1 as an example. For low aerosol concentrations in KK, the electric field smoothly decreases with altitude to its asymptotic value. In contrast, however, in the case of $Z=10^{11} \mathrm{~m}^{-3}$, as shown in figure $1(\mathrm{a})$, the electric field decreases only in the first few millimeters and then increases sharply in the lowest $\sim 2 \mathrm{~m}$ to attain its asymptotic value. The asymptotic value of electric field is inversely proportional to $\chi$ (as shown in $\mathrm{KK}$ ) and may attain a value greater than the surface electric field.

The thickness of the electrode layer is much smaller in the case of $Z=10^{11} \mathrm{~m}^{-3}$ than in the case of lower aerosol concentrations of KK. It is also smaller (figure 1a) in comparison with both, the height in which electric field increases, and the thickness of the superimposed convection current layer which is defined as the vertical extent of the steady-state value (Willett 1983). This occurs due to the drastic decrease in the life-time of ions when the aerosol concentration is large. Moreover, while the reverse electrode effect is not observed in KK when $\chi=30$, it manifests itself even for such values of $\chi$ when aerosol concentration is large which causes a large reduction in the total 

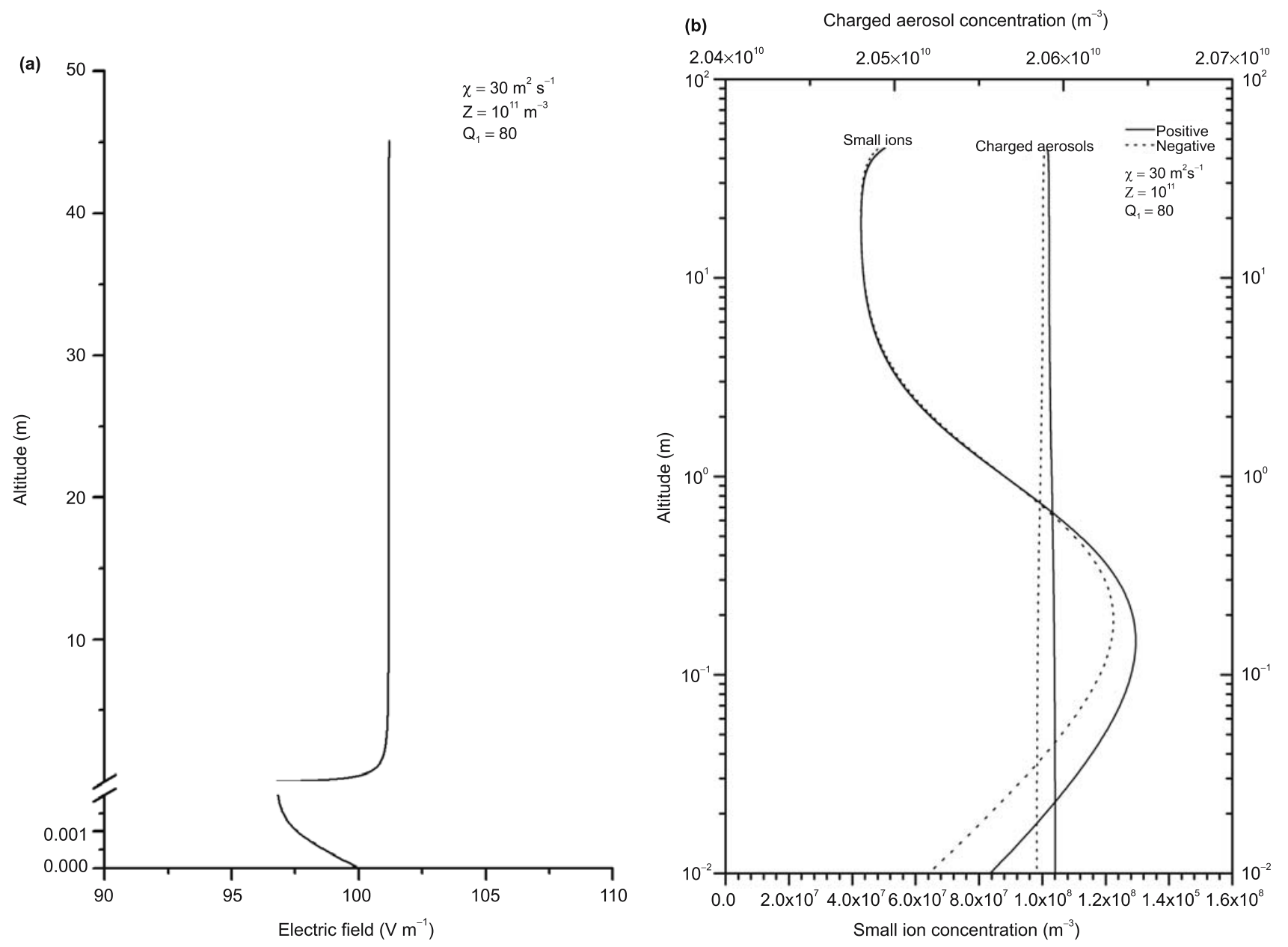

Figure 1. (a) Vertical profiles of electric field and (b) small ion concentration and charged aerosol concentration for $Z=10^{11} \mathrm{~m}^{-3}$ and $\chi=30 \mathrm{~m}^{2} \mathrm{~s}^{-1}$.

air-earth current density. So, from the results presented here and in KK, one can conclude that the increase in field in the lowest few meters of atmosphere occurs due to the intense ionization and the subsequent formation of the negative space charge layer close to surface in stable conditions, and due to the high aerosol concentrations in turbulent conditions.

In figure 1(b) we present profiles of small ion concentrations. In this figure we see that up to $1 \mathrm{~m}$ or so there is contribution of positive space charge by small ions as well as aerosol particles. Above $1 \mathrm{~m}$, the positive space charge is due to aerosol particles only. This distinction is not there in the earlier results of KK when the aerosol concentration is low. So we can conclude that in the case of maritime environment or over less polluted land surface, the electrode effect positive space charge is due to small as well as large ions at all levels in electrode layer but in the case of polluted land, it is due to aerosols only at the higher levels.

\subsection{A Method to estimate the eddy diffusion coefficient}

Hoppel and Gathman (1971) suggest that for a uniform aerosol concentration over sea surface, the value of eddy diffusion coefficient can be estimated from the measurements of the surface electric field and the small ion space charge densities at 1 or $1.5 \mathrm{~m}$ height. For low aerosol concentrations of $5 \times 10^{8}$ or $5 \times 10^{9} \mathrm{~m}^{-3}$, calculations of KK show that the large ion space charge density varies linearly with the surface electric field and does not show any significant change with the change in surface radioactivity for the same strength of turbulent mixing. Also, the vertical distribution of ion densities for different ionization profiles is such that the average space charge density in the lowest two meters or so does not differ significantly even for two extreme values of $Q_{1}$ (4.8 and 80) used in our calculations. Since the space charge density has linear relationship with the change in electric field irrespective of the total aerosol concentration, we can use our present results for high aerosol 


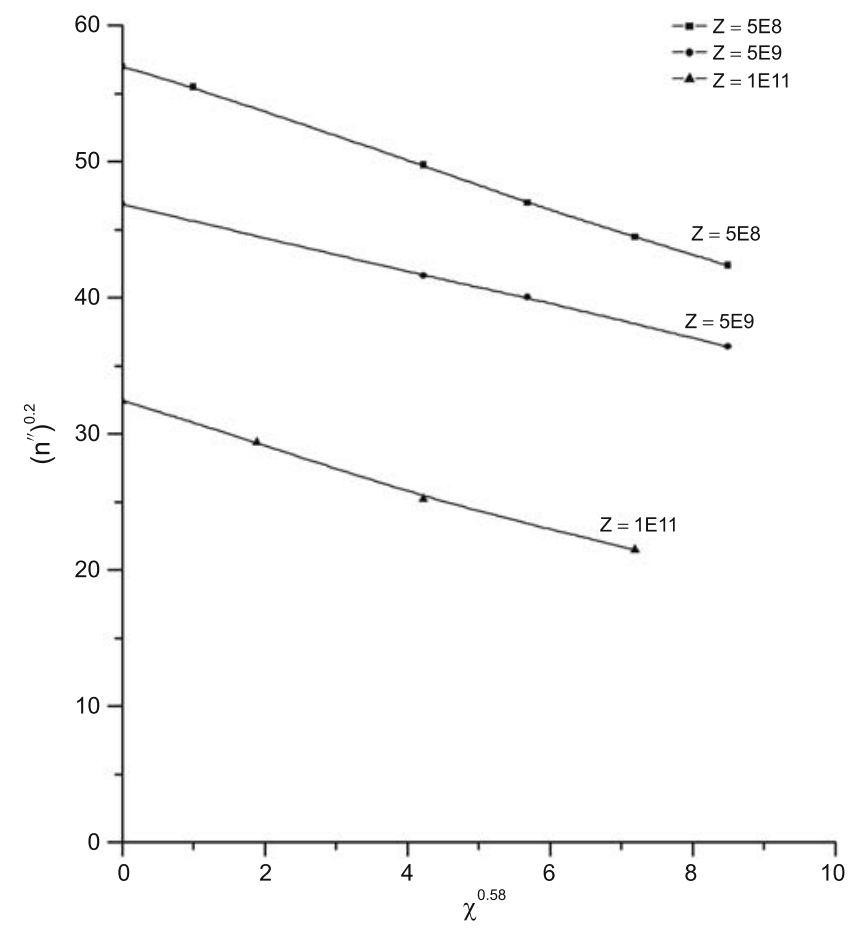

Figure 2. Variation of the $\left(n^{\prime \prime}\right)^{0.2}$ with $\chi^{0.58}$ for different values of $Z$.

concentration along with the previous results for low aerosol concentrations. Further, the space charge density close to the Earth's surface can be scaled up by dividing it with a normalized electric field, i.e.,

$$
n^{\prime \prime}=\frac{n^{\prime}}{\left|\frac{E(0)}{E}\right|},
$$

where $n^{\prime}$ is the average number density of elementary charges in the lowest $2 \mathrm{~m}, E(0)$ is the surface electric field and $E=-100 \mathrm{Vm}^{-1}$ is the electric field used for normalizing it.

A plot of space charge against $\chi$ in an aerosol environment gives a polynomial of higher degree. However, we find by hit and trial method that when $\left(n^{\prime \prime}\right)^{0.2}$ is plotted against $\chi^{0.58}$ for a fixed aerosol concentration, the curve is sufficiently linearized with a correlation coefficient for a linear fit of $>0.99$. Figure 2 shows different lines drawn from our earlier (KK) and present calculations for different values of aerosol concentrations when $E=E(0)=-100 \mathrm{~V} \mathrm{~m}^{-1}$ and $Q_{1}=80$. The lines in figure 2 for a fixed value of $Z$, can be represented by

$$
\left(n^{\prime \prime}\right)^{0.2}=A \chi^{0.58}+\left(n^{\prime}\right)_{\text {at } \chi=0}^{0.2} .
$$

The value of constant $A$ varies in the range $-1.5044 \pm 0.2576$ for aerosol concentrations

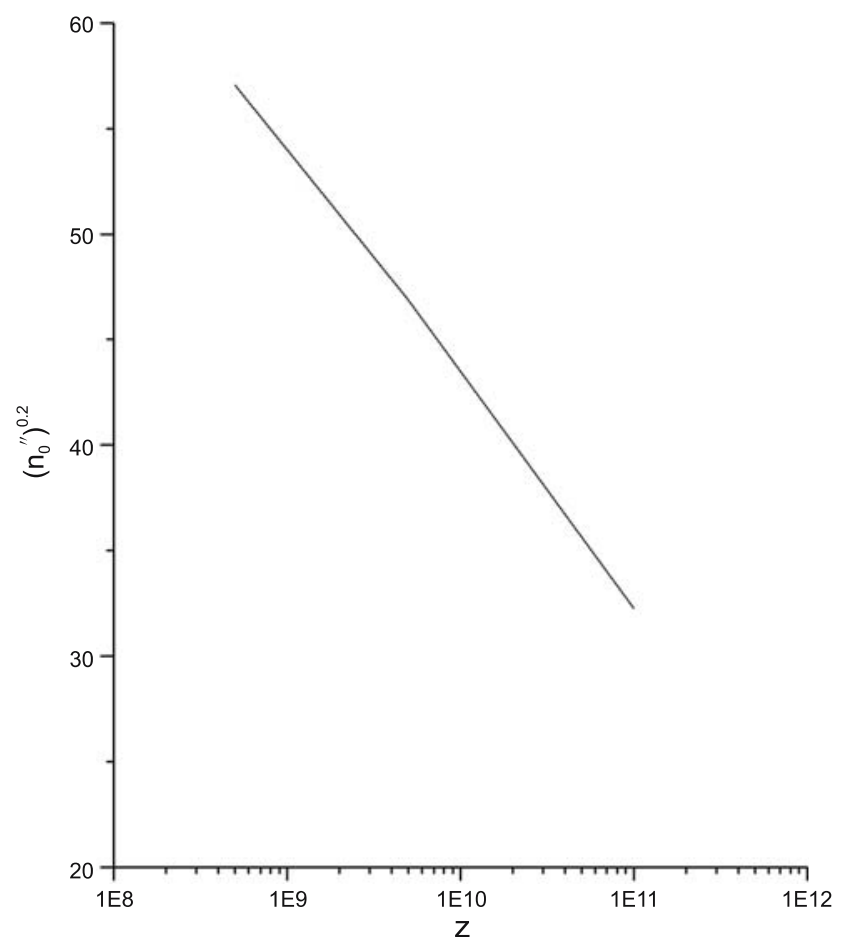

Figure 3. Variation of the $\left(\left(n^{\prime \prime}\right)_{\chi=0}\right)^{0.2}$ with $Z$ for $\chi=0$.

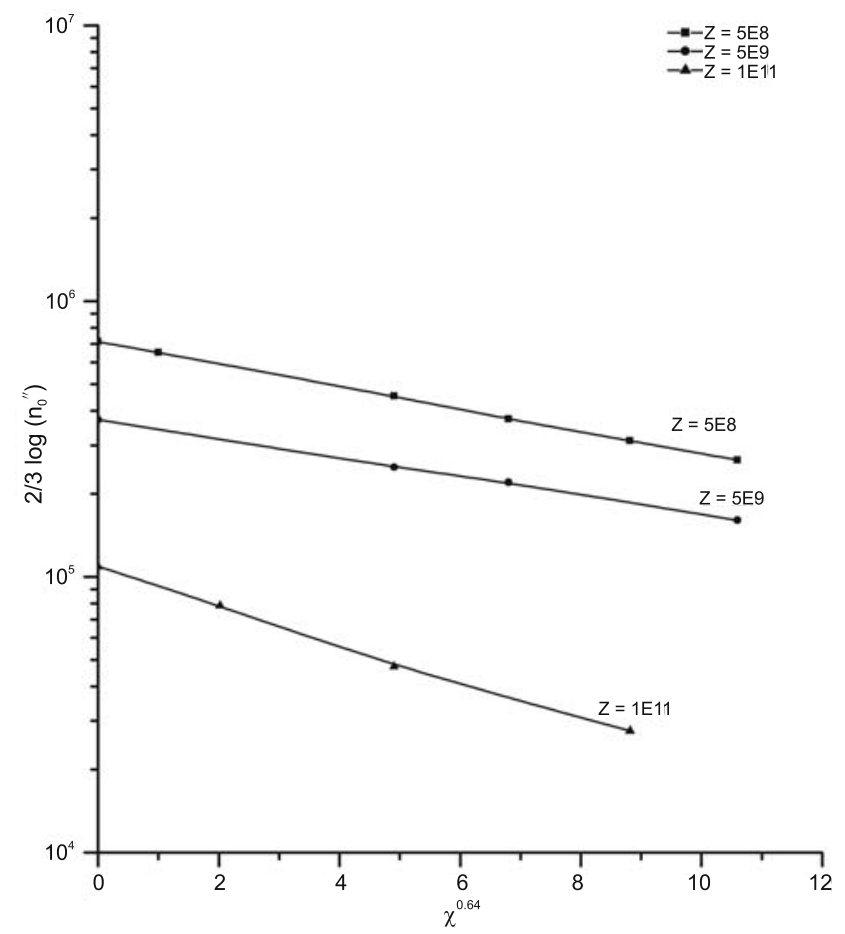

Figure 4. Variation of the $\log \left(n^{\prime \prime}\right)^{2 / 3}$ with $\chi^{0.64}$ for different values of $Z$.

between $5 \times 10^{8}$ and $10^{11} \mathrm{~m}^{-3}$. Since the slopes of lines for aerosol concentrations of $5 \times 10^{8}, 5 \times 10^{9}$ and $10^{11} \mathrm{~m}^{-3}$ are nearly the same, such variations for other values of aerosol concentrations within this range can be justifiably represented by interpolating the lines nearly parallel to these lines. 


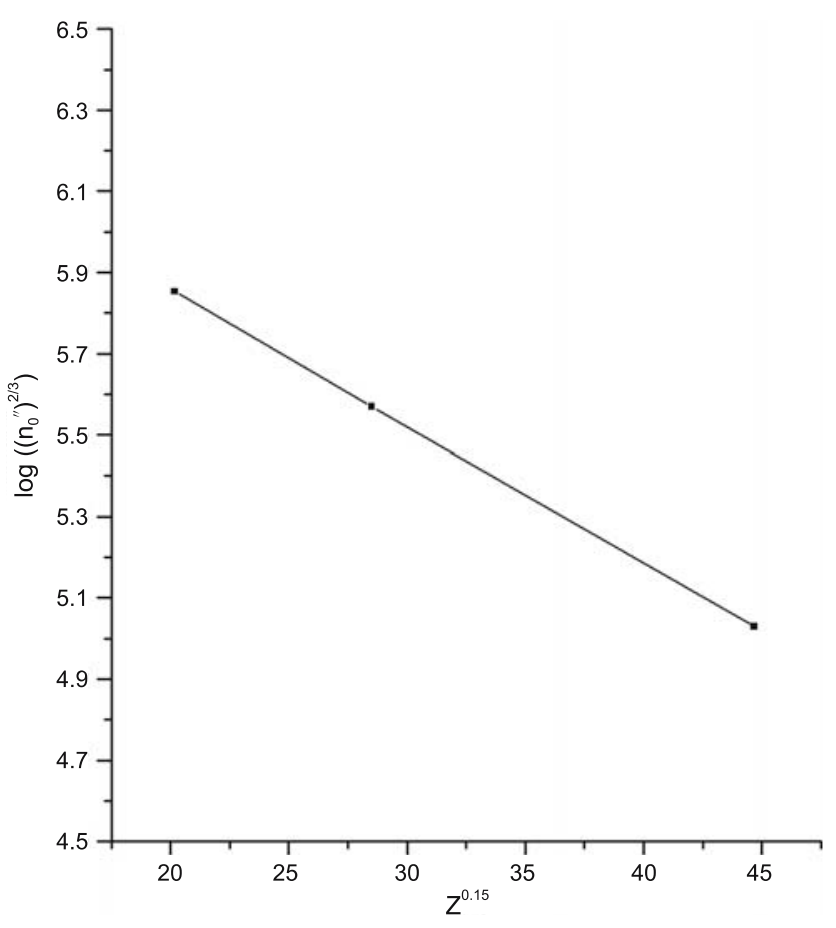

Figure 5. Variation of the $\log \left(\left(n^{\prime \prime}\right)_{\chi=0}\right)^{2 / 3}$ with $Z^{0.15}$ for $\chi=0$.

From figure 2 we can plot $\left(n^{\prime \prime}\right)^{0.2}$ against $Z$ for a fixed value of $\chi$. For example, figure 3 shows a plot for $\chi=0$. The relation is almost linear and, for a given value of $Z$, can be represented by,

$$
\left(n_{a t \chi=0}^{\prime \prime}\right)^{0.2}=-10.8066 \log _{10} Z+151.3034 .
$$

Values of constants are given up to four decimal places to have better accuracy from $\log$ functions. A similar linear relationship is observed and nearly parallel lines as in figure 2 are obtained for different values of $Z$ when $(2 / 3) \log n^{\prime \prime}$ is plotted against $\chi^{0.64}$ (figure 4 ). In this case as well the correlation coefficient for linear fit is observed $\geq 0.99$. The set of lines in figure 4 for a fixed value of $Z$ can be represented by

$$
\frac{2}{3} \log _{10} n^{\prime \prime}=B \chi^{0.64}+\frac{2}{3} \log _{10}\left(n^{\prime \prime}\right)_{\text {at } \chi=0}
$$

The value of constant $B$ varies in the range $-0.0477 \pm 0.0160$ for aerosol concentrations of $5 \times 10^{8}$ to $10^{11} \mathrm{~m}^{-3}$. Figure 5 is a plot of $\left[(2 / 3) \log _{10}\left(n^{\prime \prime}\right)\right.$ at $\left.\chi=0\right]$ against $Z^{0.15}$ for $\chi=0$ and the relation can be represented by

$$
\frac{2}{3} \log _{10}\left(n^{\prime \prime}\right)_{\text {at } \chi=0}=-0.0342 Z^{0.15}+6.5432
$$

Substituting the values of $n^{\prime \prime}$ for $\chi=0$ and a fixed value of $Z$ from (30) and (32) into (29) and (31) respectively, we get

$$
\begin{aligned}
\left(n^{\prime \prime}\right)^{0.2}= & -1.5044 \chi^{0.58}-10.8066 \log _{10} Z \\
& +151.3034 \\
\frac{2}{3} \log _{10} n^{\prime \prime}= & -0.0477 \chi^{0.64}-0.0342 Z^{0.15} \\
& +6.5432 .
\end{aligned}
$$

Equations (33) and (34) can be solved to get the values of $\chi$ and $Z$ using known value of $n^{\prime \prime}$.

Since two unknown variables, $\chi$ and $Z$, are involved, it is difficult to calculate individual error in the estimation of both. The utility of this method can, however, be checked by comparing the estimates made with this method with those made with some other independent standard method.

Table 1. Comparison of the eddy diffusion coefficients calculated from Hoppel and Gathman's (1972) data with the present $\left(K_{a e}\right)$, Hoppel and Gathman's $\left(K_{a e}(H G)\right)$ and the bulk aerodynamic $\left(K_{b a}\right)$ methods.

\begin{tabular}{lccccc}
\hline Date & $\begin{array}{c}K_{a e} \\
\left(\mathrm{~m}^{2} \mathrm{~s}^{-1}\right)\end{array}$ & $\begin{array}{c}K_{a e}(\mathrm{HG}) \\
\left(\mathrm{m}^{2} \mathrm{~s}^{-1}\right)\end{array}$ & $\begin{array}{c}K_{b a} \\
\left(\mathrm{~m}^{2} \mathrm{~s}^{-1}\right)\end{array}$ & $K_{a e}^{(H G)} / K_{b a}$ & $K_{a e} / K_{b a}$ \\
\hline 26 February & 0.06 & 0.11 & 0.103 & 1.1 & 0.58 \\
27 February & 0.05 & 0.11 & 0.083 & 1.3 & 0.60 \\
1 March & 0.11 & 0.15 & 0.105 & 1.5 & 1.05 \\
6 March & 0.09 & 0.16 & 0.120 & 1.3 & 0.75 \\
8 March & 0.14 & 0.17 & 0.123 & 1.4 & 1.14 \\
Average of 5 days & 0.09 & 0.14 & 0.107 & 1.32 & 0.82 \\
\hline
\end{tabular}

$K_{a e}$ is the mean of the two values calculated from the day's maximum and minimum values of the data of Hoppel and Gathman (1972). 


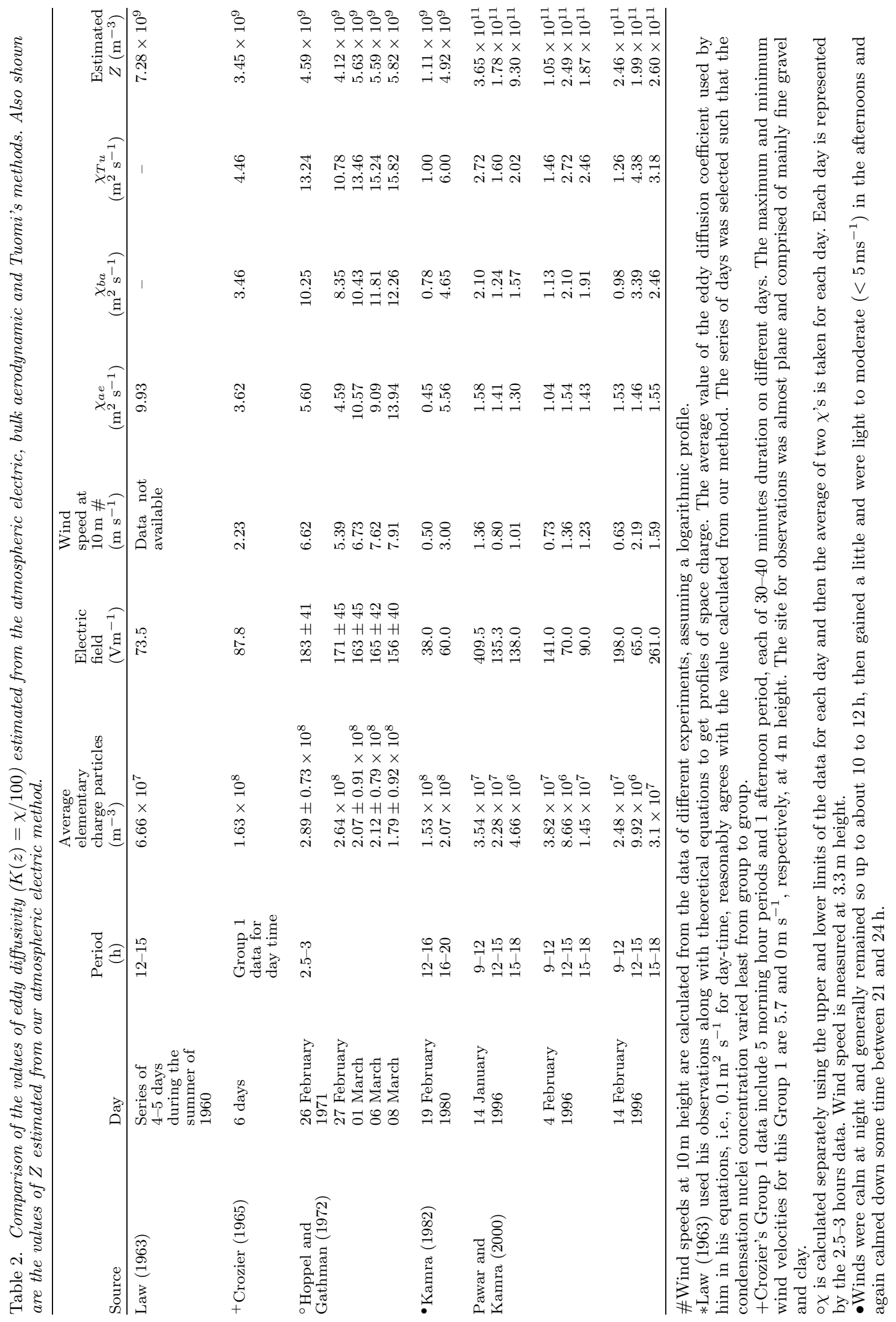


Values of the eddy diffusion coefficient in the atmosphere vary over 3 orders of magnitude under different stability conditions (Draxler 1979; Hanna 1982). Draxler (1979) finds that the standard deviation about the annual average of eddy diffusivity is about $50 \%$ for each stability category and the seasonal values all fall within this range. Since the maximum error in calculating the eddy diffusion coefficient by the atmospheric electric method may not exceed 30\% (Hoppel and Gathman 1972), such estimates may not lead to wrong conclusions in deciding the class of stability of the atmosphere.

Value of $n^{\prime \prime}$ is found from the measured values of electric field and space charge and the corresponding values of $\chi$ and $Z$ are calculated from (33) and (34).

\subsection{Comparison of the eddy diffusion coefficient values computed from different methods}

We have calculated the eddy diffusion coefficient, $K$, with our atmospheric electric method, using Hoppel and Gathman's (1972) data obtained over ocean. These data are recorded off the east coast of Barbados from two small fishing boats, $6.4 \mathrm{~m}$ in length. Wind speed is recorded by aerovanetype anemometer at $3.3 \mathrm{~m}$ level from the mean water surface along with the dew point hygrometer system for measuring air temperature and dew point. Atmospheric electrical conductivity is measured by Gerdien-type condenser. On another boat, space charge is measured using Faraday cage of $1.83 \mathrm{~m}$ in length, centered about $1.5 \mathrm{~m}$ above mean water surface. On the exterior of the cage, calibrated $\mathrm{Po}^{210}$ probe is mounted for the measurement of electric field. Table 1 displays values of eddy diffusion coefficient calculated with the atmospheric electric methods of ours $\left(K_{a e}\right)$, Hoppel and Gathman's (1972) $\left(K_{a e}(\mathrm{HG})\right)$ and with the bulk aerodynamic method $\left(K_{b a}\right)$. The average departure of $K_{a e}$ from $K_{b a}$ is $\sim 18 \%$ in our case and $\sim 32 \%$ in the case of Hoppel and Gathman (1972), i.e., with respect to $K_{a e}(\mathrm{HG})$.

Table 2 displays values of mixing parameter and aerosol concentration computed from our atmospheric electric method using data of some experiments conducted in the past. It also displays values of mixing parameter estimated from the bulk aerodynamic method and from Tuomi's formula (1982) for the average meteorological conditions prevailing during different experimental periods. In these experiments, electric field is obtained at the earth's surface, and space charge is measured either with the filtration apparatus installed at two or three levels in the lowest 1-2 m (Crozier 1965 and Kamra 1982), or with a Faraday cage with its centre at a height of either $1.5 \mathrm{~m}$ from water surface (Hoppel and Gathman 1972) or $1.8 \mathrm{~m}$ above ground (Pawar and Kamra 2000). Since space charge density has steep gradients close to ground surface during calm periods at night-time, its measurements only at three levels may not be enough for accurate estimation of its average values in the lowest $1-2 \mathrm{~m}$ during such periods. Hence no attempt has been made to evaluate the mixing parameter with the data obtained by Crozier (1965) and Kamra (1982) during such periods. Further, as shown by Pawar and Kamra (2000), the Faraday cage measurements of space charge are reliable only in the well-mixed atmosphere. Therefore, only daytime data of Pawar and Kamra (2000) are used in our comparative study. The agreement for the values of eddy diffusivity calculated from different methods in table 2 is reasonably good considering that the variables used to calculate eddy diffusivity in various methods represent an average value for a period of at least 3 hours.

\section{Conclusions}

We conclude that:

- large concentration of aerosols causes a drastic reduction in the electrode layer thickness,

- the atmospheric electrical method can be used to determine the eddy diffusion coefficient even over land surface,

- eddy diffusivity can be calculated from the measurements of surface electric field and the average space charge density in the lowest 2 meters or so, and

- the values of the eddy diffusion coefficient calculated from our atmospheric electric method reasonably agree with those determined from Hoppel and Gathman's (1972), the bulk aerodynamic, and Tuomi's methods.

\section{References}

Crozier W D 1965 Atmospheric electrical profiles below three meters; J. Geophys. Res. 70 2785-2792.

Crozier W D and Biles N 1966 Measurement of radon 220 (thoron) in the atmosphere below 50 centimeters; J. Geophys. Res. 71 4735-4741.

Draxler R R 1979 Estimating vertical diffusion from routine meteorological tower measurements; Atmos. Environ. 13 1559-1567.

Hanna S R 1982 Review of atmospheric diffusion models for regulatory Applications; W.M.O. Tech. Note No. 177.

Hess V F and O'Donnel G A 1951 On the rate of ion formation at ground level and at one meter above ground; J. Geophys. Res. 56(4) 557-562.

Holton J R 1972 An introduction to dynamic meteorology (New York: Academic Press) 319 pp.

Hoppel W A 1967 Theory of the electrode effect; J. Atmos. Terr. Phys. 29 709-721. 
Hoppel W A 1969 Electrode effect: Comparison of theory and measurement in Planetary Electrodynamics (eds) Coroniti S C and Hughes J, vol. 2, 503 pp., Gordon and Breach, Newark, N.J.

Hoppel W A and Gathman S G 1971 Determination of eddy diffusion coefficient from atmospheric electrical measurements; J. Geophys. Res. 76 1467-1477.

Hoppel W A and Gathman S G 1972 Experimental determination of the eddy diffusion coefficient over the open ocean from atmospheric electric measurements; J. Oceanogr. 2 248-254.

Kamra A K 1982 Fair weather space charge distribution in the lowest two meters of the atmosphere; J. Geophys. Res. 87 4257-4263.

Kulkarni M and Kamra A K 2001 Vertical profiles of atmospheric electric parameters close to ground; J. Geophys. Res. 106 28209-28221.

Law J 1963 The ionization of the atmosphere near the ground in fair weather; Q. J. R. Meteorol. Soc. 89 107-121.
Markson R R and Sedlacek J 1981 Turbulent transport of electric charge in the marine atmospheric boundary layer; J. Geophys. Res. 86 12,115-12,121.

Pawar S D and Kamra A K 2000 Comparative measurements of the atmospheric electric space charge density made with the filtration and Faraday cage techniques; Atmos. Res. 54 105-116.

Stull R B 1988 An introduction to boundary layer meteorology (New York: Springer) $680 \mathrm{pp}$.

Tuomi T J 1982 The atmospheric electrode effect over snow; J. Atmos. Terr. Phys. 44 737-745.

Willett J C 1978 An analysis of the electrode effect in the limit of strong turbulent mixing; J. Geophys. Res. $\mathbf{8 3}$ 402-408.

Willett J C 1979 Fair Weather Electric Charge Transfer by Convection in an Unstable Planetary Boundary Layer; J. Geophys. Res. 84 703-718.

Willett J C 1983 The turbulent electrode effect as influenced by interfacial ion transfer; J. Geophys. Res. $\mathbf{8 8}$ 8453-8469. 